BMC

Genomics

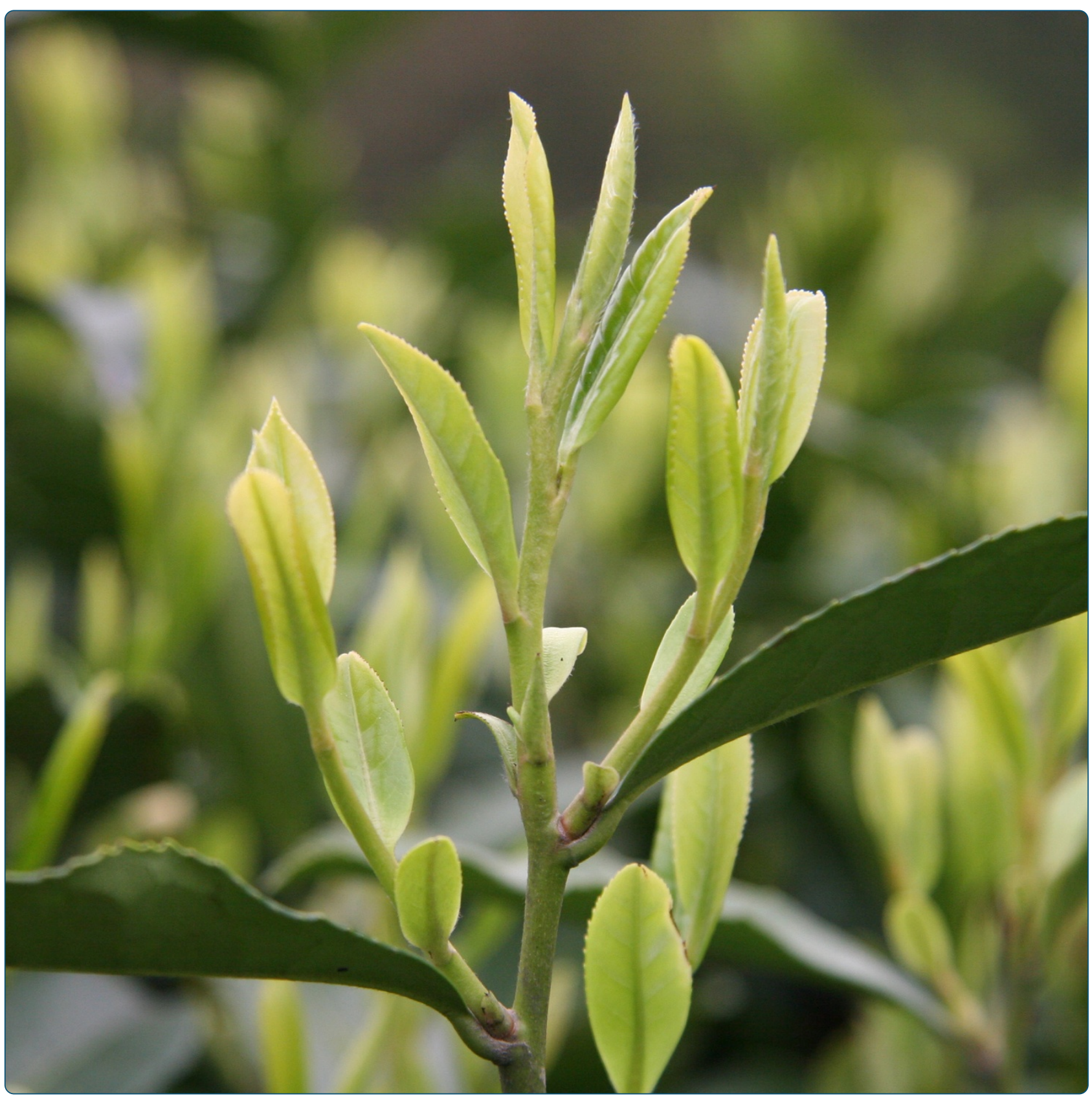

Global transcriptome profiles of Camellia sinensis during cold acclimation

Wang et al. 


\title{
Global transcriptome profiles of Camellia sinensis during cold acclimation
}

\author{
Xin-Chao Wang ${ }^{1+}$, Qiong-Yi Zhao ${ }^{2,3+}$, Chun-Lei Ma ${ }^{1}$ Z Zong-Hong Zhang ${ }^{3}$, Hong-Li Cao ${ }^{1}$, Yi-Meng Kong ${ }^{2}$, \\ Chuan Yue ${ }^{1}$, Xin-Yuan Hao ${ }^{1}$, Liang Chen ${ }^{1}$, Jian-Qiang Ma ${ }^{1}$, Ji-Qiang Jin ${ }^{1}$, Xuan $\mathrm{Li}^{2^{*}}$ and Ya-Jun Yang ${ }^{1 *}$
}

\begin{abstract}
Background: Tea is the most popular non-alcoholic health beverage in the world. The tea plant (Camellia sinensis (L.) O. Kuntze) needs to undergo a cold acclimation process to enhance its freezing tolerance in winter. Changes that occur at the molecular level in response to low temperatures are poorly understood in tea plants. To elucidate the molecular mechanisms of cold acclimation, we employed RNA-Seq and digital gene expression (DGE) technologies to the study of genome-wide expression profiles during cold acclimation in tea plants.

Results: Using the Illumina sequencing platform, we obtained approximately 57.35 million RNA-Seq reads. These reads were assembled into 216,831 transcripts, with an average length of $356 \mathrm{bp}$ and an N50 of $529 \mathrm{bp}$. In total, 1,770 differentially expressed transcripts were identified, of which 1,168 were up-regulated and 602 down-regulated. These include a group of cold sensor or signal transduction genes, cold-responsive transcription factor genes, plasma membrane stabilization related genes, osmosensing-responsive genes, and detoxification enzyme genes. DGE and quantitative RT-PCR analysis further confirmed the results from RNA-Seq analysis. Pathway analysis indicated that the "carbohydrate metabolism pathway" and the "calcium signaling pathway" might play a vital role in tea plants' responses to cold stress.
\end{abstract}

Conclusions: Our study presents a global survey of transcriptome profiles of tea plants in response to low, non-freezing temperatures and yields insights into the molecular mechanisms of tea plants during the cold acclimation process. It could also serve as a valuable resource for relevant research on cold-tolerance and help to explore the cold-related genes in improving the understanding of low-temperature tolerance and plant-environment interactions.

Keywords: Camellia Sinensis, Cold Acclimation, RNA-Seq, DGE, Genome-wide Expression Profiles, Tea Plants

\section{Background}

Low temperatures are one of the most important environmental factors that temperate plants have to cope with during their life cycles. Some plants can enhance their freezing tolerance after exposure to low but non-freezing temperatures for a period of time, a process known as cold acclimation (CA) [1]. CA is a complex process that involves cellular, physiological, metabolic and molecular

\footnotetext{
* Correspondence: lixuan@sippe.ac.cn; yjyang@mail.tricaas.com

${ }^{\dagger}$ Equal contributors

${ }^{2}$ Key Laboratory of Synthetic Biology, Institute of Plant Physiology and Ecology, Shanghai Institutes for Biological Sciences Chinese Academy of Sciences, Shanghai 200032, China

'Tea Research Institute, Chinese Academy of Agricultural Sciences; National Center for Tea Improvement, Key Laboratory of Tea Plant Biology and Resources Utilization, Ministry of Agriculture, Hangzhou 310008, China Full list of author information is available at the end of the article
}

modifications. When plants sense the cold temperature, a series of protective mechanisms are triggered [2-4]. These include resetting the cellular framework; alternating the composition, structure and function of the plasma membrane; synthesizing cryoprotectant molecules such as soluble sugars, sugar alcohols and low-molecular-weight nitrogenous compounds; decreasing the ratio of free water content; improving the scavenging activity of reactive oxygen species (ROS); and introducing antifreeze proteins. These alterations help plants maintain a metabolic balance of substance and energy in cold environments. A group of cold-related genes has been reported to regulate these aforementioned changes [2-7]. Moreover, changes in gene expression have been demonstrated to occur during CA in a wide range of plant species, and hundreds of cold inducible genes have been identified [8].

\section{Biomed Central}


Tea is the most popular non-alcoholic health beverage in the world, and the tea plant (Camellia sinensis (L.) O. Kuntze) is one of the most important economic crops in China, India, Sri Lanka, Kenya, among others [9]. As an evergreen woody plant, the tea plant can be grown in tropical to subtropical climates. Due to the local climate changes, tea plants have to cope with low temperatures during the wintertime. Low temperatures are one of the most critical environmental factors that limit its growth, survival and geographical distribution [10]. Thus, finding ways to improve tea plants' resistance to low temperatures is of great importance. Like other perennial evergreen woody crops, during the CA process, the cold tolerance of tea plants enhances with the decrease in temperature and reduces with the increase in temperature. A previous study showed that when the average air temperature decreases to around $7{ }^{\circ} \mathrm{C}$, tea plants undergo the CA process, and after the average air temperature increases to over $9^{\circ} \mathrm{C}$, tea plants start the de-acclimation process [11]. There are few studies that have focused on the cellular, physiological and metabolic changes during $\mathrm{CA}$ in tea plants. When tea plants undergo the CA process, the thickness of palisade tissue is increased and the stability of plasma membrane is enhanced. In addition, the concentration of the cytochylema and ratio of bound water in the cytoplasm, the amount of unsaturated fatty acids and total proteins in the plasma membrane, and the content of soluble proteins in the leaf are also increased. Meanwhile, the activities of some detoxification enzymes, such as catalase (CAT), superoxide dismutase (SOD), peroxidase (POD) and esterase (EST) are increased, whereas the metabolic activity is decreased $[11,12]$. Some cold-induced genes have been cloned in tea plants $[13,14]$. As a complex biological phenomenon, the ability of tea plants to resist the cold is regulated by a series of genes involved in a complex regulatory network [15]. Using an 'omics' research strategy to understand the mechanism of CA in tea plants is the key to improving tea productivity and geographical distribution.

RNA-Seq is a recently developed approach using a massively parallel sequencing strategy to generate transcriptome profiles. It has emerged as a cost-effective approach for high-throughput sequence determination and has unprecedentedly improved the efficiency and speed of gene discovery $[16,17]$. Digital gene expression (DGE) is a tag-based sequencing approach according to which short tags are generated by endonuclease. The expression level of genes in the sample is measured by counting the number of tags generated from each transcript [18]. This study demonstrates the first attempt to use a combination of RNA-Seq and DGE to study the transcriptome profiles in tea plants and thereby gain a deeper insight into the molecular mechanism of CA. The resulting transcriptome profiles from tea plants not only contributes to the in-depth knowledge of the genes involved in CA but also improves our understanding of plant-environment interactions.

\section{Results and discussion}

Cold tolerance changes in tea plant during the CA process Cold tolerance in tea plants varies under different temperatures and can be monitored by the relative electrical conductivity using an electrolyte leakage assay. Figure 1 shows a complete course of the CA process for a natural temperature change period from December 2010 to March 2011. Before December 1, the average outdoor temperature (for five days) was above $10^{\circ} \mathrm{C}$, and the relative electrical conductivity of tea-plant leaves (CK) was at $\sim 100 \%$, indicating that the tea plant has a low level of cold tolerance. After the tea plant underwent a period of time at relatively low temperatures $\left(<10^{\circ} \mathrm{C}\right)$, its relative electrical conductivity decreased, and the cold tolerance of the tea plant is enhanced. When temperatures decreased to their lowest point, the relative electrical conductivity also reached its lowest level with the cold tolerance being at the highest level (CA1). Afterwards, the temperature rose and when the average temperature reached above $10^{\circ} \mathrm{C}$, the relative electrical conductivity increased to over $80 \%$ and then maintained at a high level. The tea plant was subsequently de-acclimated (CA3), and its cold tolerance was weak (Figure 1).

To obtain the transcriptomic response to the cold environment during the CA process, we selected tea-plant leaves from three stages, non-acclimated (CK), fully acclimated (CA1) and de-acclimated (CA3) for RNA-Seq and digital gene expression (DGE) studies.

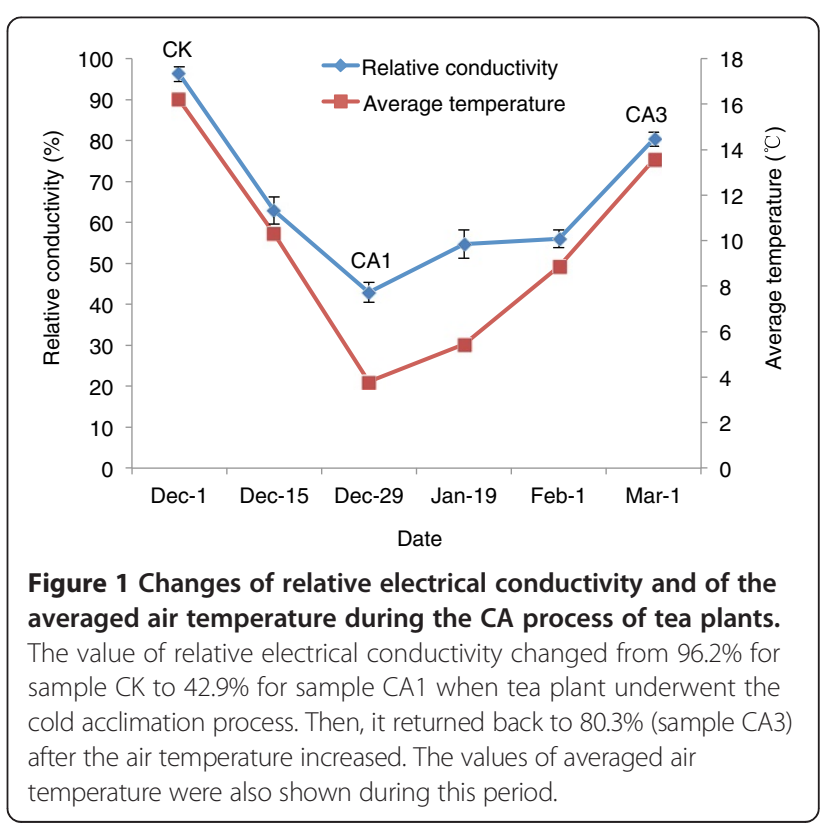




\section{RNA-Seq and de novo assembly}

We performed RNA-Seq analyses for CA1, CA3 and CK using the Illumina HiSeq2000 genome analyzer. Totally, 57.35 million paired-end reads with a read length of $90 \mathrm{bp}$ were generated (hereafter referred to as dataset 1 , Table 1 ). Since low-quality nucleotides from the ends of reads may lead to incorrect assembly outputs [19], we trimmed the low-quality or ambiguous nucleotides at both ends of the reads. De novo assembly was performed with the trimmed reads using Trinity [20]. Trinity was specially developed for de novo assembly from short-read RNA-Seq data, which has been shown to be the best single $k$-mer assembler $[19,20]$. In total, 226,026 transcripts were reconstructed. After removing the redundant transcripts caused by small variations as described in the previous study [19], a final set of 216,831 transcripts were obtained. The average transcript size is $356 \mathrm{bp}$, and the N50 is $529 \mathrm{bp}$ (Table 2).

The transcriptome of $C$. sinensis was reported in a previous study by Shi et al. [21]. They produced RNA-Seq data from the mixed tissues of $C$. sinensis using Illumina GA IIx (hereafter referred to as dataset 2). A combination of dataset 1 and dataset 2 was also generated, which we called dataset 3, representing all available RNA-Seq data for $C$. sinensis. Short reads of dataset 2 and dataset 3 were pre-processed by the procedure described above, and then used separately for de novo assembly. The assembly outcome from dataset 1 attains the longest average read length and N50, while that from dataset 3 yields the most number of transcripts and total base pairs (Table 2). In order to evaluate the efficiency of short-read usage during the de novo assembly, we mapped our RNA-Seq reads back to three sets of reconstructed transcripts, respectively. Transcripts produced from dataset 1 achieved the best performance, with the highest mapping ratio for our short reads (Table 2). More than $10 \%$ of the short reads failed to be aligned if only dataset 2 was used for the de novo assembly, indicating that previous transcriptome sequences of $C$. sinensis are far from saturated. Although more transcriptome sequences could be produced from de novo assembly using dataset 3 than dataset 1 , the mapping ratio could not be improved (Table 2), indicating that the additional transcripts from dataset 3 are most likely transcripts that are expressed in tissues other than the leaves of tea plants. Thus these additional transcripts are unable to contribute to this study. Based on this scenario, we chose the transcripts from dataset 1 to carry out the downstream analysis.

Table 1 Summary for RNA-Seq datasets of $C$. sinensis

\begin{tabular}{lcccc}
\hline & CK & CA1 & CA3 & Total \\
\hline Number of reads (million) & 17.54 & 13.65 & 26.16 & 57.35 \\
Total bases (raw, Gb) & 1.58 & 1.23 & 2.35 & 5.16 \\
Total bases (trimmed, Gb) & 1.50 & 1.16 & 2.30 & 4.96 \\
\hline
\end{tabular}

Table 2 Summary for the outcomes of de novo transcriptome assembly using three datasets in C. sinensis

\begin{tabular}{lccc}
\hline & Dataset 1 & Dataset 2 & Dataset 3 \\
\hline Number of transcripts $(\geq 100 \mathrm{bp})$ & 226,026 & 177,439 & 295,551 \\
Number of transcripts & 216,831 & 170,650 & 282,395 \\
(remove redundancy) & & & \\
Total base pairs (Mbp) & 77.36 & 50.36 & 94.71 \\
Average length (bp) & 356 & 295 & 335 \\
N50 (bp) & 529 & 403 & 480 \\
Mapping ratio (\%) & 76.94 & 64.87 & 76.91 \\
\hline
\end{tabular}

\section{Functional annotation of $C$. sinensis transcriptome}

To predict and analyze the function of the assembled transcripts, non-redundant sequences were submitted to a BLASTx (E-value $\leq 10^{-5}$ ) search against the following databases: the NCBI's NR database, UniRef90 [22], the Arabidopsis Information Resource (TAIR, version 10), Kyoto Encyclopedia of Genes and Genomes (KEGG, version 58) [23] and Clusters of Orthologous Groups from 7 eukaryotic complete genomes (KOG) [24]. We found that about one third of all non-redundant transcripts had significant homology with genes in either the NR or UniRef90 databases (Table 3).

Arabidopsis thaliana is one of the most well-studied dicot plants, with a complete reference genome and comprehensively annotated gene sequences. A BLAST search against genes from Arabidopsis produced more definitive annotations and helped us to evaluate the quality and coverage of our assembled transcripts. It is notable that 16,882 Arabidopsis genes located uniformly on five chromosomes were covered by 60,392 transcripts (Tables 3 and 4).

A BLAST analysis of the assembled transcripts against the KEGG database showed that 21,194 transcripts were annotated with corresponding Enzyme Commission (EC) numbers and assigned to the reference canonical KEGG pathways (Table 3). A search against the KOG database reported that 41,341 transcripts had the best hits when the E-value was less than or equal to $10^{-5}$. Since some transcripts could be assigned multiple KOG functions, altogether 46,291 functional annotations were produced and all hit transcripts were grouped in 25 categories (Tables 3 and 5).

In total, 72,967 transcripts got the best hits with known proteins in at least one of the five databases and 16,430 transcripts had similarity to proteins in all of the five databases (Figure 2, Table 3).

To functionally categorize the assembled transcripts, gene ontology (GO) [24] terms were assigned to each transcript based on the best BLASTx hit from the NR database using Blast2GO [25]. Out of 71,289 transcripts with NR annotation, 30,115 transcripts were assigned 80,176 GO term annotations in three main GO 
Table 3 Summary for the BLASTx results of $C$. sinensis transcriptome against five databases

\begin{tabular}{lc}
\hline & Sequences $(\mathbf{n})$ \\
\hline Transcripts & 216,831 \\
Number of transcripts & 716,831 \\
BLASTx against NR & 71,709 \\
BLASTx against UniRef90 & 60,392 \\
BLASTx against TAIR10 & 21,194 \\
BLASTx against KEGG & 41,341 \\
BLASTx against KOG & 72,967 \\
All annotated transcripts & 16,430 \\
Transcripts hit all five databases & \\
Unigenes & 179,753 \\
Number of unigenes & 51,883 \\
BLASTx against NR & 52,217 \\
BLASTx against UniRef90 & 42,969 \\
BLASTx against TAIR10 & 15,086 \\
BLASTx against KEGG & 29,356 \\
BLASTx against KOG & 53,201 \\
All annotated unigenes & 11,388 \\
Unigenes hit all five databases &
\end{tabular}

categories including biological process, cellular component and molecular function (Table 3, Additional file 1). If a gene contained some conserved domains, the domain information would be useful for interpreting the gene's function. To annotate the potential domains inside the reconstructed sequences, the open reading frame (ORF) was predicted for each transcript (Methods), and then all transcripts with predicted ORF were used to search against the Pfam database based on profile hidden Markov model methods. In total, 41,599 transcripts were assigned Pfam domain information and were categorized into 4,504 domains/families. Most domains/families were found to contain a small number of transcripts (Figure 3A). According to the frequency of the occurrence of $C$. sinensis transcripts contained in each Pfam domain, Pfam domains/families were ranked and the top 10 abundant domains/families are listed in Figure 3B, with hit results similar to the previous study [21]. Among these domains/families, "Protein kinase domain" and its subclass "Protein tyrosine kinase" are known to regulate the majority of cellular pathways. Proteins with "leucine-rich repeats" domain are known to be frequently involved in the formation of protein-protein interactions, and "PPR repeat" has been reported to be a large protein family in plants with versatile functions [26]. Moreover, the "NB-ARC" protein family, comprised of resistance proteins, was highly represented. Other protein families, such as "reverse transcriptase" and "RNA recognition motif", which have some basic functions in plants, were also found in the top ten of the list.

Trinity produced all potential alternative spliced isoforms during the de novo assembly, and isoforms originated from the same gene locus were assumed to share the same "chrysalis component", "butterfly sub-component" and some of the paths in the de Bruijn graph. We chose the longest transcript in each locus to get the unigene set, resulting in 179,753 unigenes (Table 3). Potential isoforms in each locus reported by Trinity would be useful in array/ primer design for quantitative gene expression and future alternative splicing analyses.

\section{Identification of genes involved in cold acclimation}

The abundance estimation for reconstructed transcripts was determined by RSEM software package that was shown to have the ability to effectively use ambiguouslymapping reads and to accurately estimate isoform-level abundance for de novo assembled transcripts without reference [27]. The DESeq package [28] and the winflat program were then applied to identify differentially expressed genes. CA-related genes were identified based on the fold change on the abundance of each gene and the corresponding false discovery rate, which resulted in 1,770 differentially expressed genes. Of these, 1,168 were upregulated and 602 were down-regulated (Additional file 2), indicating that more genes were activated than repressed during the CA process. Dozens of cold-regulated or coldrelated genes were found in this differential expression list, including cold sensor or signal transduction genes, coldresponsive transcription factor genes, plasma membrane stabilization related genes, osmosensing-responsive genes and detoxification enzymes genes.

\section{Cold sensor or signal transduction genes}

The signal transduction pathway plays a pivotal role in the response to the stress of low temperatures [29]. It is well known that $\mathrm{Ca}^{2+}$ acts as a key messenger in regulating growth and developmental processes and plays a crucial role in stress signaling, i.e. cold stress [30]. Cold stress could activate $\mathrm{Ca}^{2+}$ channels to increase the cytosolic $\mathrm{Ca}^{2+}$ level, and then trigger phospholipase $\mathrm{C}$ and $\mathrm{D}$, producing inositol

Table 4 Distribution of BLASTx hit genes against Arabidopsis TAIR10

\begin{tabular}{ccccccccc}
\hline Chromosome & Chr1 & Chr2 & Chr3 & Chr4 & Chr5 & ChrC & ChrM & Total \\
\hline Total genes & 9,263 & 5,560 & 6,908 & 5,356 & 8,089 & 88 & 122 & 35,386 \\
No. of hits & 4,374 & 2,445 & 3,293 & 2,600 & 4,080 & 36 & 54 & 16,882 \\
$\%$ & $47.22 \%$ & $43.97 \%$ & $47.67 \%$ & $48.54 \%$ & $50.44 \%$ & $40.91 \%$ & $44.26 \%$ & $47.71 \%$ \\
\hline
\end{tabular}


Table 5 KOG functional classification of $C$. sinensis transcripts

\begin{tabular}{|c|c|c|}
\hline Description & Code & Transcript (n) \\
\hline \multicolumn{3}{|l|}{1 Information storage and processing } \\
\hline Translation, ribosomal structure and biogennesis & $J$ & 1,812 \\
\hline RNA processing and modification & A & 1,729 \\
\hline Transcription & K & 2,341 \\
\hline Replication, recombination and repair & L & 1,194 \\
\hline Chromation structure and dynamics & B & 706 \\
\hline \multicolumn{3}{|l|}{2 Cellular processing and signaling } \\
\hline $\begin{array}{l}\text { Cell cycle control, cell division, } \\
\text { chromosome partitioning }\end{array}$ & $\mathrm{D}$ & 1,091 \\
\hline Nuclear structute & Y & 274 \\
\hline Defense mechanisms & V & 385 \\
\hline Signal transduction mechanisms & $\mathrm{T}$ & 4,836 \\
\hline Cell wall/membrane/envelope biogenesis & M & 558 \\
\hline Cell motility & $\mathrm{N}$ & 24 \\
\hline Cytoskeleton & Z & 1,072 \\
\hline Extracellular structure & W & 187 \\
\hline $\begin{array}{l}\text { Intracellular trafficking, secretion, and } \\
\text { vesicular transport }\end{array}$ & $U$ & 2,289 \\
\hline $\begin{array}{l}\text { Posttranslational modification, } \\
\text { protein turnover, chaperones }\end{array}$ & $\mathrm{O}$ & 4,152 \\
\hline \multicolumn{3}{|l|}{3 Metabolism } \\
\hline Energy production and conversion & C & 1,800 \\
\hline Carbohydrate transport and metabolism & G & 2,387 \\
\hline Amino acid transport and metabolism & $E$ & 1,514 \\
\hline Nucleotide transport and metabolism & $\mathrm{F}$ & 432 \\
\hline Coenzyme ransport and metabolism & $\mathrm{H}$ & 343 \\
\hline Lipid ransport and metabolism & I & 1,440 \\
\hline Inorganic ion ransport and metabolism & $\mathrm{P}$ & 1,159 \\
\hline $\begin{array}{l}\text { Secondary metabolites biosynthesis, } \\
\text { transport and catabolism }\end{array}$ & Q & 1,428 \\
\hline \multicolumn{3}{|l|}{4 Poorly characterized } \\
\hline General function prediction only & $\mathrm{R}$ & 11,108 \\
\hline Function unknown & S & 2,030 \\
\hline
\end{tabular}

triphosphate and phosphatidic acid, respectively. Inositol triphosphate could further amplify $\mathrm{Ca}^{2+}$ signatures, and phosphatidic acid is proposed as a membrane-based secondary messenger molecule $[31,32]$. Subsequently, many signaling pathways are triggered, such as $\mathrm{Ca}^{2+}$-dependent protein kinases (CDPKs), mitogen-activated protein kinases (MAPKs), calcineurin B-like protein (CBL), calmodulin, etc. Doherty et al. [33] and Yang et al. [34] found that the calmodulin-binding transcription activator (CAMTA) and a novel calcium/calmodulin-regulated receptor kinase (CRLK1) were crucial for cold tolerance in plants. $\mathrm{Ca}^{2+}$ influx into the cell was considered to occur upstream of the expression of CBFs and COR genes in the cold signaling

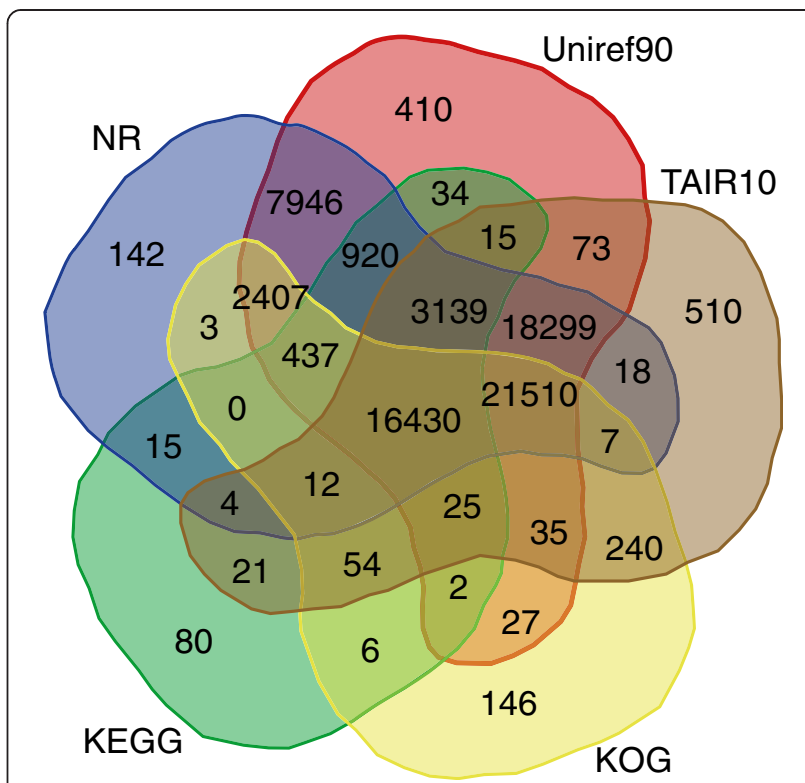

Figure 2 Venn diagram showing the BLAST results of $C$. sinensis transcriptome against five databases. De novo reconstructed transcript sequences were used to BLAST search against public databases including NCBI's NR, UniRef90, TAIR10, KEGG and KOG. The number of transcripts that have significant hits (E-value $\leq 10^{-5}$ ) against the five databases is shown in each intersection of the Venn diagram.

pathway [35,36]. In this study, 13 genes, which were annotated as CDPKs, CBL, calmodulin, CAMTA, MAPK and phospholipase, were identified as being involved in signal transduction upon low temperature stress. Among these genes, 9 (4 calmodulin genes, 2 CDPK genes, 1 CAMTA gene and 2 phospholipase genes) were up-regulated in CA1, whereas 4 (1 phospholipase gene, 1 calmodulin gene, 1 CBL gene and 1 MAPK gene) were down-regulated.

Plant protein kinases belong to a large superfamily, some of which have been known to play a central role in cellular signaling, for example CDPKs and MAPKs. In addition, a growing body of evidence has shown that receptor-like kinases (RLKs) are involved in the perception of environmental signals $[37,38]$. Histidine kinases (HKs), being localized to the cellular membranes and endoplasmic reticulum, are the major signaling molecules and are involved in the two-component signaling pathways that mediate plant-sensed environmental signals and regulate the downstream environmental stress response $[39,40]$. In this study, 27 RLKs genes and 2 HKs genes were differentially expressed and all of these were up-regulated in CA1 samples, which indicates that protein kinases play an important role in the CA process in tea plants.

\section{Cold-responsive transcription factor genes}

Transcription factors (TFs) play important functions in plant development and stress tolerance [35]. Fifty-eight genes encoding putative TFs in C. sinensis were identified. 

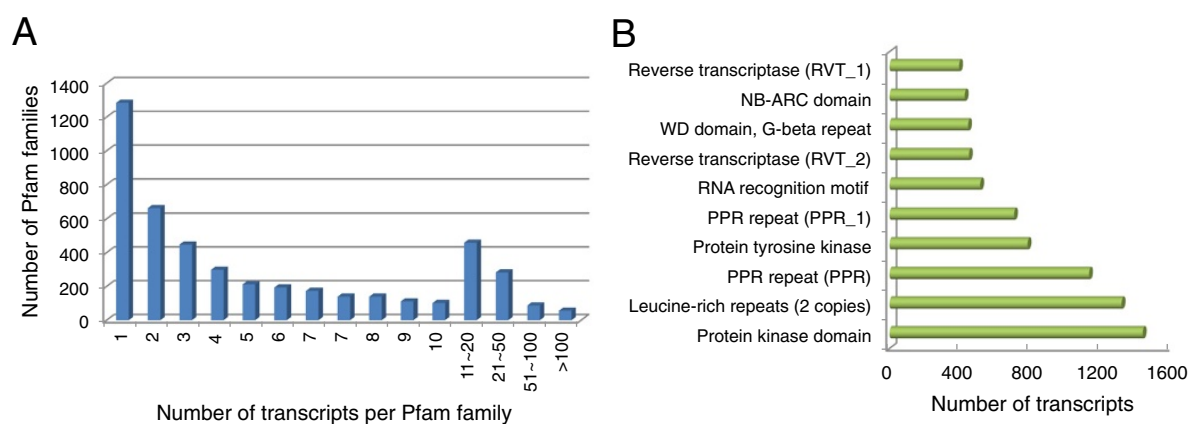

Figure 3 Protein families in C. sinensis transcriptome. Shown are the number of Pfam domains/families versus the occurrence of $C$. sinensis transcripts contained in each domain/family (A), and the 10 most abundant protein families (B) in C. sinensis.

These TFs could be divided into 9 groups (AP2/ERF, bHLH, WRKY, MYB, NAC, bZIP, heat shock, GARS and zinc finger protein) based on the classification of their Arabidopsis homologs, and most of them have been reported to be linked to cold stress resistance in plants [41-49]. Among these TFs, 37 genes were up-regulated and 21 genes were down-regulated in our CA1 sample.

Of the 9 groups of TFs, zinc finger was the most enriched TF family, containing 31 genes of the 58 cold-responsive TFs, with 18 genes being up-regulated and 13 being down-regulated. There were 5 genes in the bHLH family (4 up- and 1 down-regulated), 5 genes in the MYB family (4 up- and 1 down-regulated), 5 genes in the WRKY family ( 3 up- and 2 down-regulated) and 3 genes in the NAC family ( 1 up- and 2 down-regulated). In addition, 2 genes in the bZIP family, 3 genes in the GARS family and 2 genes encoding heat shock proteins were all upregulated, while 2 genes in the AP2/ERF family were down-regulated in the CA1 sample. It is interesting to find down-regulated genes in the AP2/ERF family, as these suggest that the interaction of light and temperature is of special importance for plants during the CA process. Catalá et al. [50] and Jurczyk et al. [51] have also reported that light is required for full CA in Arabidopsis and Festuca pratensis.

\section{Genes related to the stabilization of the plasma membrane and osmosensing-responsiveness}

The plasma membrane is believed to be a primary site of injury from freezing in plants. The process of CA can stabilize the membrane structure and prevent it from damage [52]. Under freezing temperatures, membranes must be kept fluid in order to sustain the functional activity of membrane proteins and membranes themselves [53]. Alterations occur in the composition of proteins and lipids (e.g. increases in the unsaturation level of the membrane lipids) in the plasma membrane in response to $\mathrm{CA}$, and these are associated with an increase in freezing tolerance $[53,54]$. In our study, we identified 3 lipid-transfer protein (LTP) genes and 1 fatty acid desaturase (FAD) gene. Among these, 2 LTP genes and a FAD gene were up-regulated and 1 LTP gene was down-regulated. These genes were known to regulate the level of unsaturated fatty acids, and then to further mediate the regulation of membrane fluidity [55-57].

Moreover, in order to maintain the structural stabilization of the plasma membrane during the CA process, some proteins function as inhibitors to regulate the activity of ice nucleators. These proteins are so-called anti-freezing proteins (AFPs), such as $\beta$-1, 3-glucanase-like proteins (GLPs), chitinase-like proteins (CLPs), thaumatin-like proteins (TLPs), polygalacturonase inhibitor proteins (PGIPs) and late-embryogenesis-abundant proteins (LEAs). In the CA1 sample, more genes encoding these proteins were up-regulated compared with genes in non-acclimated samples $[36,58]$. In our study, we found 7 AFP-related genes, including 4 CLPs, 1 TLP, 1 PGIP and 1 LEA that were upregulated in the CA1 sample, indicating that during the $C A$ process, tea plants became able to tolerate freezing temperatures through the enhancement of membrane stability.

The stabilization of the plasma membranes is also related to the osmotic equilibrium. In order to maintain osmotic balance, plants accumulate a range of compatible solutes, including soluble sugars (saccharose, trehalose, rafinose), sugar alcohols (ribitol, inositol, sorbitol), and low-molecularweight compounds (such as proline, glycine betaine, glutamic acid) as cryoprotectant molecules in response to cold stress [59-61]. Accordingly, the expression of these metabolism related genes also changes during CA $[29,36,62]$. We identified 13 genes related to the carbohydrate metabolic pathway from 1,770 differentially expressed genes, including 4 galactosidases ( 3 up- and 1 down-regulated), 5 amylases (all up-regulated), 1 galactinol synthase (up-regulated), 1 raffinose synthase (up-regulated) and 2 trehalose-6-phosphate synthases (all up-regulated). These genes are key genes of the carbohydrate metabolic pathway, and are closely involved with the CA process [63]. Three monosaccharide transporter genes (2 up- and 1 down-regulated) were identified as well. Monosaccharide transporters play an important role in sugar transport and 
distribution in plants. The expression of monosaccharide transporter genes is also regulated by cold stress [64]. These results suggested that the carbohydrate metabolic pathway plays a critical role in tea plants during the CA process.

\section{Validation of RNA-Seq results by DGE and qRT-PCR}

Digital gene expression (DGE) library sequencing was performed to validate the cold-regulated transcripts identified by RNA-Seq. In our study, three DGE libraries were sequenced: CA1, CA3 and CK, for which 3.69, 3.62 and 3.68 million raw tags were generated, respectively (Table 6). After removing low-quality tags, the total number of clean tags per library ranged from 3.53 to 3.60 million. Clean tags from three DGE libraries were mapped onto our assembled transcriptome sequences. Up to $24.25 \%$ of transcripts were detected by DGE tags (Table 6).

Of the 1,770 differentially expressed transcripts identified by RNA-Seq, 1,460 were detected by DGE sequencing, but 870 were mapped by uncertain tags (tags that mapped to at least two different transcripts) and another 192 transcripts did not have enough tags ( $<1$ 'tags per million' (TPM) counts for all three samples) to differentiate expressions among CA1, CA3 and CK samples. This result illustrates that DGE sequencing was limited to identify differential expression across the full scale of transcriptome profiles, especially for genes with paralogs or multiple isoforms that shared the same tags (CATG+17bp). Of the remaining 398 transcripts, the majority of them (376/398) showed consistent expression patterns between DGE and RNA-Seq, with the corresponding Pearson's $r$ being 0.77 and 0.81 for CA1/ CA3 and CA1/CK, respectively (Figure 4, Additional file 3), demonstrating the degree of consistency between DGE and RNA-Seq platforms.

It is worth noting that some transcripts, though not many, showed different expression patterns in the profiling results from RNA-Seq and DGE (Figure 4). Determining which method is more robust and why the two approaches

Table 6 Summary for DGE datasets

\begin{tabular}{lccc}
\hline Summary & CA1 & CA3 & CK \\
\hline Raw tags & $3,689,000$ & $3,615,500$ & $3,675,000$ \\
Clean tags (total) & $3,599,673$ & $3,528,122$ & $3,586,229$ \\
Aligned tags & $2,027,613$ & $2,402,599$ & $1,810,760$ \\
Aligned tags (\%) & $56.33 \%$ & $68.10 \%$ & $50.49 \%$ \\
Clean Tags (unique) & 882,038 & 456,976 & 932,551 \\
Aligned unique-tags & 117,591 & 104,604 & 111,505 \\
Aligned unique-tags (\%) & $13.33 \%$ & $22.89 \%$ & $11.96 \%$ \\
Transcripts with aligned tags & 52,590 & 46,331 & 51,333 \\
Transcripts with aligned tags (\%) & $24.25 \%$ & $21.37 \%$ & $23.67 \%$ \\
Transcripts with uniquely aligned tags & 33,380 & 28,462 & 32,519 \\
Transcripts with uniquely aligned tags (\%) & $15.39 \%$ & $13.13 \%$ & $15.00 \%$ \\
\hline
\end{tabular}

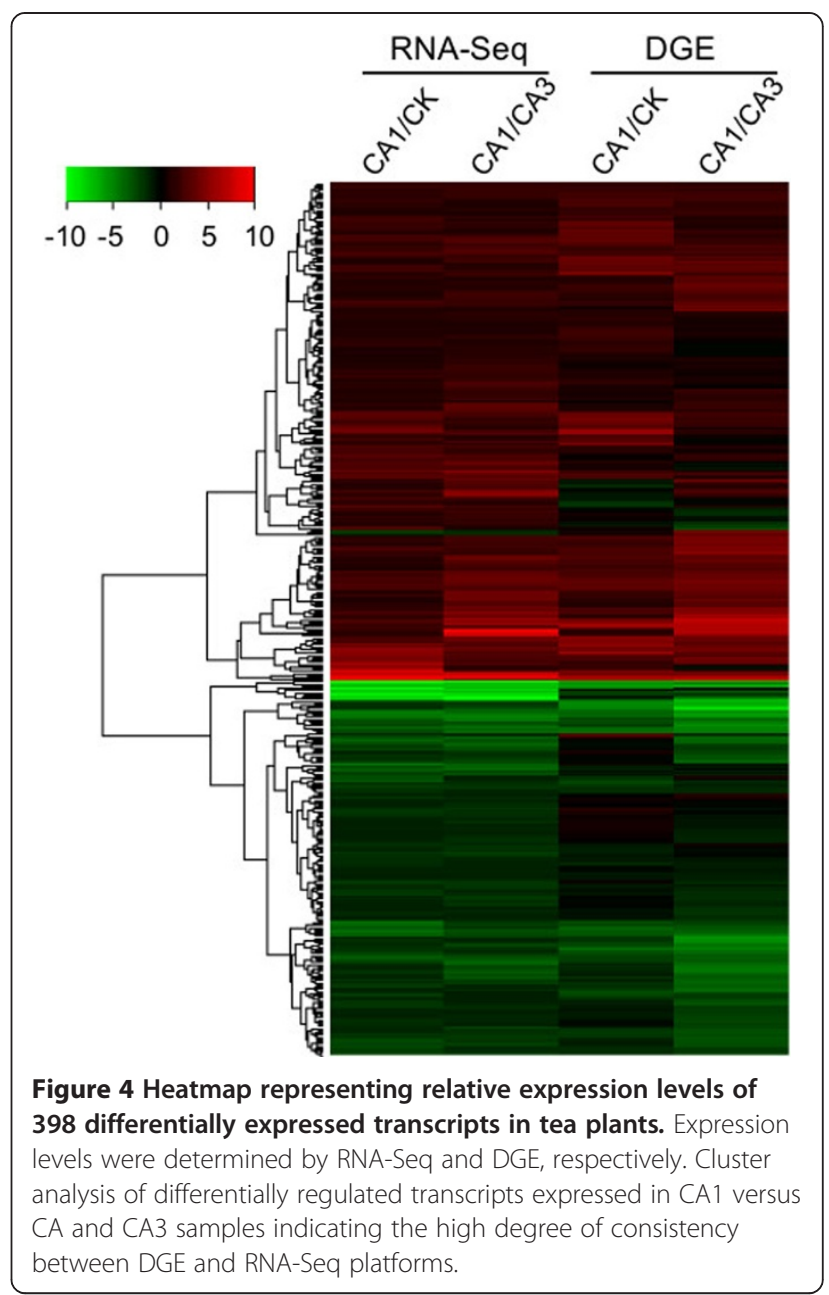

yield different results would be useful for identifying the correct outcomes in this study and for other researchers to choose the appropriate approach in their future studies. To address this, 10 of these transcripts that showed inconsistent results from RNA-Seq and DGE platforms were randomly selected to assess their relative expression patterns among CK, CA1 and CA3 using quantitative RT-PCR approach (qRT-PCT). For most of these (8/10), similar expression patterns were observed compared with those from RNA-Seq results, while in the other 2 transcripts there were only partial consistencies with either RNA-Seq or DGE results (Additional file 4). In general, RNA-Seq outperforms DGE based on the results from these 10 cases. The less accurate estimation of the gene expression level by DGE approach could be due to some unknown reason(s) or to the fact that the same tags may exist in other transcripts that were partially reconstructed after de novo transcriptome assembly and lack the complete tag sequences. Since the DGE approach counts all tags to the transcript with the exactly matched tag sequences, this may result in the incorrect estimation of the expression level for some transcripts. In the remaining two genes, inconsistent 
expression patterns were observed among the results from the three approaches. These genes expressed at relatively variable levels may be affected by factors other than a cold environment and these kinds of false positives could be largely avoided if more biological replicates were included.

The DGE method is widely applied for studying the transcriptome. However, it has limitations in presenting a global view of transcriptome profiles. It is powerless to detect the abundance of transcripts when 1) there is no CATG site in transcripts or 2) several transcripts share the same tag, situations involving two unrelated genes, paralogs, or alternatively spliced isoforms. Both closely related paralogs and alternatively spliced isoforms might exhibit various spatial and temporal expression patterns, or even have different functions. Thus, the ability to correctly estimate isoform expression levels will be necessary for understanding complicated biological mechanisms.

To further test the reliability of outcomes produced from the next generation sequencing platform, quantitative RT-PCR (qRT-PCR) analysis was performed for 18 of the 1,770 differentially expressed transcripts. These 18 transcripts were manually selected as representatives for their potential roles in cold tolerance according to their annotations. The expression patterns of 17 genes detected by qRT-PCR fit well with those from RNA-Seq results, with one annotated as a basic helix-loop-helix DNA-binding superfamily protein being inconsistent (Figure 5).

\section{Pathways involved during the $\mathrm{CA}$ process in $\mathrm{C}$. sinensis}

The 1,770 transcripts were used to search the KEGG pathway to determine whether the genes involved in CA were from specific pathways. In total, 200 pathways were identified, 20 of which were significantly enriched during CA $(P<0.05$, Additional file 5). Of these significantly enriched, metabolism was the largest category (99 transcripts), including carbohydrate metabolism (28), glycan biosynthesis and metabolism (21), energy metabolism (15), amino acid metabolism (14), metabolism of terpenoids and polyketides (9), enzyme families (5), xenobiotics biodegradation and metabolism (4) and lipid metabolism (2). Moreover, calcium signaling pathway (9) and membrane transport pathway (7) were enriched as well. Numerous studies reported that carbohydrate metabolism plays an important role during the CA process [65-69]. In this study, metabolic pathways for carbohydrates stood out from the enrichment analysis, including pathways for 49 differentially expressed transcripts ( 28 for carbohydrate metabolism and 21 for glycan biosynthesis and metabolism), indicating that the regulation of carbohydrates is crucial for tea plants during CA.

Previous studies have shown that calcium acts as a pivotal mediator in the signal transduction pathway during the CA process $[29,36]$. Calcium is a secondary messenger in plant signaling processes, and calcium/calmodulin-mediated signaling is believed to play an important role in plants during the cold stress response [30,34,70]. In this study, the calcium signaling pathway was also enriched, and most of the genes in this pathway were up-regulated in the CA1 sample, proving the importance of the calcium signaling pathway for the tea plants' response to cold stress.

\section{Conclusions}

In this study, we present a global survey for transcriptome profiles in tea plants during the CA process using RNA-Seq and DGE. A large number of genes from tea plants involved in diverse biological or molecular pathways were identified during the CA process, such as genes involved in cold signal sensors or transduction, genes related to the stabilization of plasma membranes, osmosensing-responsive genes, and stress-responsive transcription factor genes. A diagram is shown to illustrate tea plants' responses to low temperatures during the CA process (Figure 6). The results showed that a series of complex regulatory networks were triggered in tea plants during CA. Our study provides insights into the molecular mechanisms of tea plants during the CA process. It could also serve as a valuable resource for relevant research on cold-tolerance and help to explore the cold-related genes in improving the understanding of low-temperature tolerance and plantenvironment interactions.

\section{Methods}

\section{Low-temperature tolerance assays and RNA preparation}

The tea plant cultivar 'Camellia sinensis (L.) O. Kuntze cv. Longjing 43' was planted in the China National Germplasm Hangzhou Tea Repository (CNGHTR) at the Tea Research Institute, Chinese Academy of Agricultural Sciences (TRI, CAAS). Starting in October 2010, intact mature leaves were selected in every 10-15 days until March 2011, when the average temperature became higher than $15^{\circ} \mathrm{C}$. All samples were washed with distilled deionized water and divided into two parts, one for $-80^{\circ} \mathrm{C}$ storage using liquid nitrogen for quick freezing and the other for evaluating low-temperature tolerance using an electrolyte leakage assay. RNAprep pure Plant Kit (Tiangen, Beijing, China) was used for total RNA extraction, and Agilent 2100 Bioanalyzer was used to test the RNA integrity with a minimum integrity value of 8 .

The low-temperature tolerance was determined from leaf samples by electrolyte leakage assay similar with previous study [71]. Briefly, leaves were washed with deionized water. Leaf samples $(5 \mathrm{~mm}$ in diameter) were extracted using a hole puncher and the midvein of the leaf was excluded. Leaf samples $(0.5 \mathrm{~g})$ were placed in closed vials containing $20 \mathrm{ml}$ of deionized water and incubated at $25^{\circ} \mathrm{C}$ on a rotary shaker for $24 \mathrm{~h}$. Then the electrical conductivity of the solution (L1) was determined. Samples were then autoclaved at $100^{\circ} \mathrm{C}$ for $20 \mathrm{~min}$ and the final electrical 

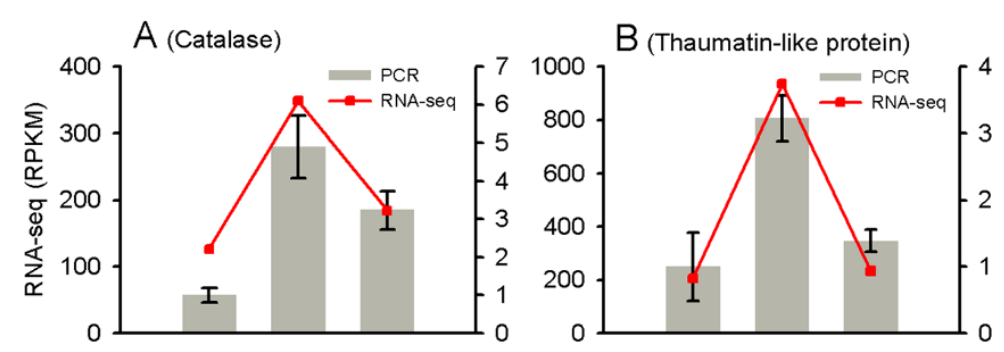

$\mathrm{C}$ (Ascorbate peroxidase)
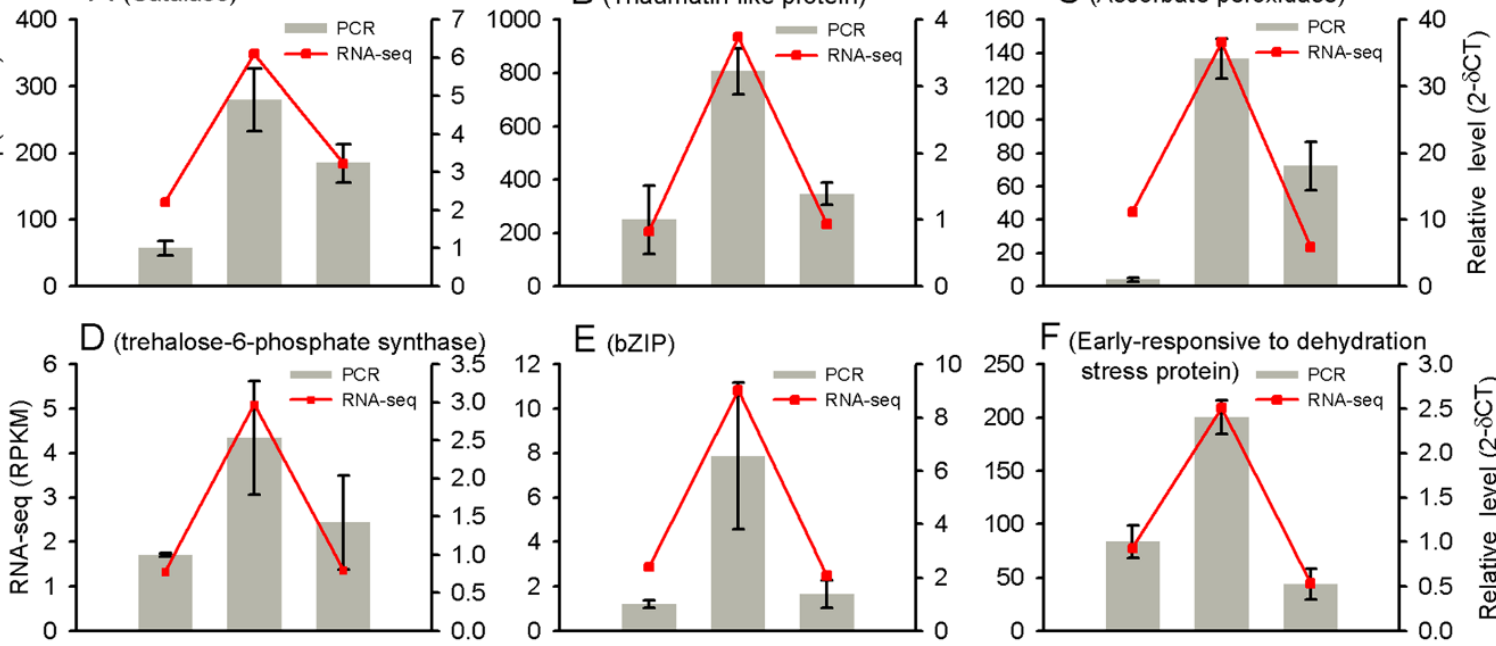

$E(b Z I P)$

$\mathrm{F}$ (Early-responsive to dehydration
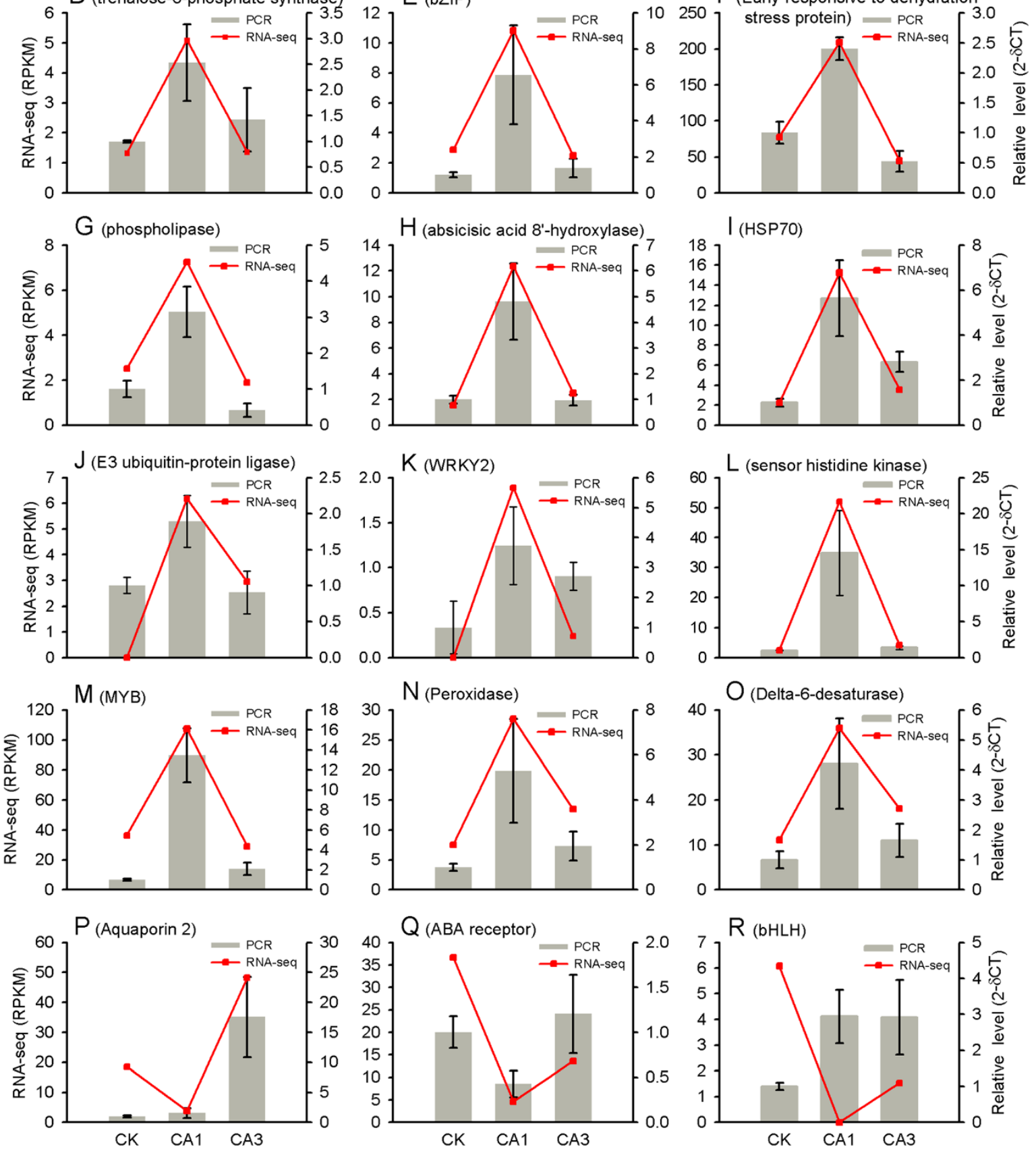

Figure 5 (See legend on next page.) 
(See figure on previous page.)

Figure 5 Quantitative RT-PCR validations. 18 genes were selected for the quantitative RT-PCR analysis, including catalase (A), thaumatin-like protein $(\mathbf{B})$, ascorbate peroxidase $(\mathbf{C})$, trehalose-6-phosphate synthase $(\mathbf{D})$, bZIP $(\mathbf{E})$, early-responsive to dehydration stress protein $(\mathbf{F})$, phospholipase (G), absicisic acid 8'-hydroxylase (H), HSP70 (I), E3 ubiquitin-protein ligase (J), WRKY2 (K), sensor histidine kinase (L), MYB (M), peroxidase $(\mathbf{N})$, delta-6-desaturase $(\mathbf{0})$, Aquaporin 2 (P), ABA receptor $(\mathbf{Q})$ and bHLH (R). The18S ribosomal gene was chosen as the reference gene.

conductivity (L2) was determined after equilibration at $25^{\circ} \mathrm{C}$. The EL (relative electrical conductivity) was defined as follows: EL $(\%)=(\mathrm{L} 1 / \mathrm{L} 2 \times 100 \%)$. Based on the level of electrolyte leakage, three samples including non-acclimated (CK, the date for sample collection was 1st December, 2010), fully acclimated (CA1, the date was 29th December, 2010) and de-acclimated (CA3, the date was 1st March, 2011) were selected for RNA-Seq and DGE analyses.

\section{Library preparation and RNA-Seq}

The samples for RNA-Seq were prepared using Illumina's kit and following manufacturer's recommendations. In short, mRNA was purified from $20 \mu \mathrm{g}$ of total RNA using oligo (dT) magnetic beads, followed by fragmentation, in which the mRNA is fragmented into small pieces using divalent cations under elevated temperature. The cleaved RNA fragments were used for first-strand cDNA synthesis using reverse-transcriptase and random primers followed by second-strand cDNA synthesis using DNA polymerase I and RNase $\mathrm{H}$. After the end repair process and ligation of adapters, the products were enriched by PCR to create the final cDNA library.

The cDNA library was sequenced from both $5^{\prime}$ and $3^{\prime}$ ends using the Illumina $\mathrm{HiSeq}^{\text {in }} 2000$ platform according to the manufacturer's instructions. The fluorescent image processing, base-calling and quality value calculation were performed by the Illumina data processing pipeline 1.4, in which 290 bp paired-end reads were obtained.

\section{Short-read RNA-Seq datasets}

In our study, we performed RNA-Seq for three samples from tea plants that represented three key stages during the CA process, including CA1, CA3 and CK. We called these dataset 1 . The accession code of our RNA-Seq dataset is SRA061043. The previous study reported the transcriptome of C. sinensis, with 75 bp paired-end reads produced from the Illumina GAII platform, and we called this dataset 2. Its accession code is SRX020193, which includes samples from seven different tissues of $C$. sinensis: tender shoots, young leaves, mature leaves, stems, young roots, flower buds and immature seeds [21]. Furthermore, we combined dataset 1

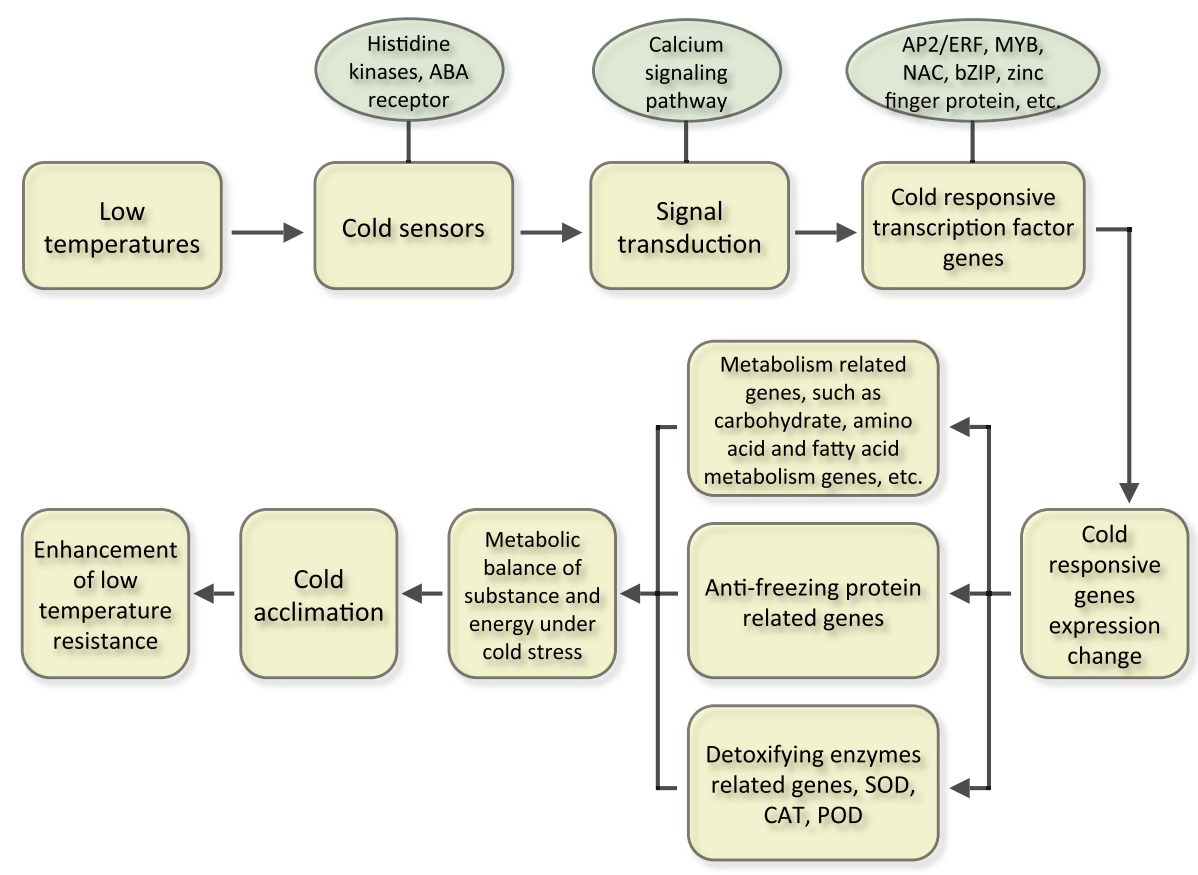

Figure 6 A diagram of tea plants' responses to low temperatures during the CA process. 
and dataset 2 together as dataset 3 in order to compare the outcomes from de novo assembly using different datasets.

\section{Preprocessing and de novo assembly}

Raw data is preprocessed before de novo assembly: lowquality nucleotides (we defined nucleotides with a quality score less than 20 as low-quality nucleotides) in the last 20 cycles and ambiguous nucleotides in the first five cycles were trimmed by custom PERL script. After preprocessing, we obtained a total of $\sim 4.96 \mathrm{G}$ bases $(\mathrm{Gb}), \sim 1.90 \mathrm{~Gb}$ and $\sim 6.86 \mathrm{~Gb}$ quality filtered short reads for dataset 1 , dataset 2 and dataset 3, respectively.

De novo assemblies for these three datasets were performed separately by Trinity (release 20110713) [20]. The command-line parameters are "-seqType fq --left 1.fq --right 2.fq --paired_fragment_length 300 --min_contig_length 100 --run_butterfly --output RNASeq_Trinity --CPU 8".

\section{Removal of redundancy}

Some isoforms reconstructed by Trinity with the same "chrysalis component" and "butterfly sub-component" had only small variations, such as SNPs, small insertions or deletions; such variations introduced redundancies for the assembly outcomes. CD-HIT-EST [72] was used to remove the shorter redundant transcripts when they were entirely covered by other transcripts with more than $99 \%$ identity. This set of transcripts was then used to count the basic assembly statistics and for downstream analysis.

\section{Gene annotation and classification}

All non-redundant transcripts ( $\geq 100 \mathrm{bp}$ ) were used to search against the NR, UniRef90 [22], TAIR10, KEGG (version 58) [23] and KOG [24] databases by BLASTALL package (release 2.2.22) with the significant threshold of E-value $\leq 10^{-5}$. Each known gene from the best BLASTx hit was parsed and assigned. Gene ontology (GO) [73] terms for each transcript were assigned based on the best BLASTx hit from the NR database using Blast2GO software (version 2.3.5) [25] with an E-value threshold of $10^{-5}$.

The ORF of assembled transcripts was determined based on the results of BLASTx search in the following order: NR, UniRef90, KEGG and KOG. Extending from both sides of the aligned region, the coding region sequences were translated into amino acid sequences with the standard codon table using custom PERL scripts. For those transcripts without any BLASTx hit against known databases, the best potential coding region was predicted using the software BestORF with parameters trained on Arabidopsis ESTs. The predicted amino sequences were submitted to search against the Pfam database (version 25.0) [74] for domain/family annotation using HMMER 3.0, with the 'Best Match Cascade' protocol. The "optimising allowed match overlap" method [75] was used to resolve complex overlapping protein domains.

\section{Mapping reads to transcripts}

In order to get assembly statistics for the ratio of number of reads that could be mapped back to transcripts (mapping ratio), bowtie (version 0.12.7) [76] was used to align short reads to the reconstructed transcripts, with parameters "-q --solexa1.3-quals --fr -1 fq1 - 2 fq2 -k 1 -v 3 -X 300". Custom PERL scripts were used to summarize the aligned results.

\section{Calculation of gene expression level}

RSEM (v1.1.11) [27] was used to quantify transcript abundance in each sample, with parameters "-phred64quals --estimate-rspd --calc-ci --out-bam --fragmentlength-min 100 --fragment-length-max 350", and then the RSEM-estimated fragment counts were fed into DESeq package (1.0.6) [28] to get the 'baseMean' value. The false discovery rate (FDR) of each comparison (CA1 vs. CA3 and CA1 vs. CK) was calculated by the winflat program which implements a rigorous statistical analysis described by Audic and Claverie [18]. The FDR $\leq 0.01$ and the absolute value of $\log 2$ ratio $\geq 1$ were used as the threshold of significant differences in gene expression. Those genes that were significantly differentially expressed in both CA1 vs. CK and CA1 vs. CA3 were identified as potentially related to CA.

\section{Digital gene expression}

Tag library preparation for three samples was performed in parallel using the Illumina gene expression sample preparation kit. Briefly, $6 \mu \mathrm{g}$ total RNA from each sample was used for mRNA capture with magnetic oligo (dT) beads. First- and second-strand cDNA were synthesized. Bead-bound cDNA was subsequently digested with NlaIII. The cDNA fragments with 3' ends were then purified with magnetic beads, and the Illumina adapter 1 was ligated to their 5 ends. The junction of the Illumina adapter 1 and CATG site is the recognition site of MmeI, which cuts the cDNA at 17 bp downstream of the CATG site, producing tags linked with adapter 1 . After removing 3' fragments with magnetic beads precipitation, the Illumina adaptor 2 was ligated to the 3 ' ends of tags. The ligation products were enriched by PCR amplification (15 cycles) and purified by $6 \%$ TBE PAGE Gel electrophoresis. Sequencing was carried out on the Illumina HiSeq 2000 platform, as recommended by the manufacturer, for 35 cycles.

Raw image data was transformed by base calling into sequence data. Adaptor sequences were removed by custom PERL scripts and low-quality tags with ambiguous nucleotide(s) were discarded. All remaining tags were then aligned to the reconstructed transcripts by bowtie with parameters "-a -f -v 0". Tags that could not be uniquely aligned were discarded. For gene expression 
analysis, the number of expressed tags was counted and then normalized to TPM.

\section{Quantitative real-time RT-PCR (qRT-PCR) analysis}

In order to validate the reliability of RNA-Seq and DGE experiments, 28 transcripts were selected for quantitative RT-PCR (qRT-PCR) test. The RNA $(1 \mu \mathrm{g})$ of each sample was treated with DNase I (Tiangen, China), then real-time PCR was performed using PrimeScript ${ }^{\mathrm{TM}} \mathrm{RT}$ reagent $\mathrm{qPCR}$ Kit fromTakara (Dalian, China) under the following parameters: $95^{\circ} \mathrm{C}$ for $30 \mathrm{~s}, 40$ cycles at $94^{\circ} \mathrm{C}$ for $15 \mathrm{~s}, 60^{\circ} \mathrm{C}$ for $34 \mathrm{~s}$. Fluorescence intensity was measured using the Applied Biosystems 7300 Sequence Detection System (Carlsbad, CA, USA). Triplicates of each reaction were performed. To ensure the robustness of the reference gene used in the qRT-PCR experiment, we analyzed the gene expression stability of 4 commonly used housekeeping genes (18S RNA, $\beta$-Actin, GAPDH and $\alpha$-Tubulin) across the cold acclimation process. As previously reported by others [77], our results also showed that the 18S RNA gene was the most stable one for its constant expression levels and was finally chosen as the reference gene in our study. The relative expression of the genes in the three samples was calculated using the $2^{-\Delta \Delta \mathrm{Ct}}$ method described earlier [78]. The result of the qRT-PCR was presented as fold changes in gene expression relative to that of CK sample. So, the relative value of $\mathrm{CK}$ is 1 and the relative values of CA1 and CA3 samples were normalized to that of CK sample. All data are shown as the mean \pm SD and all primer information is provided in Additional file 6.

\section{Additional files}

\section{Additional file 1: Gene Ontology classification of C. sinensis} transcriptome. Gene Ontology (GO) terms are summarized in three main categories: cellular component, molecular function and biological process. The left and right $y$-axes are in $\log (10)$ scale, indicating the percentage and the number of genes within a specific $\mathrm{GO}$ category, respectively.

Additional file 2: List of differentially expressed transcripts of $C$ sinensis during CA. Listed are transcript ID (column A), the length of each transcript (B), numbers of raw reads for each transcript (C-E), RPKM $(F-H)$, "baseMean" (I-K), fold change, $P$-value and FDR for CA3/CA1 (L-P) and $\mathrm{CK} / \mathrm{CA} 1(\mathrm{Q}-\mathrm{U})$, and annotations search against NR, UniRef90, TAIR10, KEGG and KOG (V-AH). "baseMean" values were generated by DESeq package. RPKM: reads per kilobase per million mapped reads.

Additional file 3: The relative gene expression values of 398 transcripts based on RNA-Seq and DGE platforms. Transcript ID (column A), relative gene expression values (log2 ratio) for CA1/CK and CA1/CA3 generated from both DGE (B-C) and RNA-Seq (D-E) are shown.

Additional file 4: Quantitative RT-PCR validations for 10 randomly selected transcripts. The quantitative RT-PCR experiment was performed on 10 randomly selected transcripts that show distinct results from RNA-Seq and DGE approaches. Consistent expression patterns between RNA-Seq and GRT-PCR results were observed in eight of these transcripts ( $A, B, C, D, E, H, I$ and J), while the other 2 transcripts were only partially consistent ( $\mathrm{F}$ and $\mathrm{G}$ ).
Additional file 5: Pathways identified in C. sinensis during CA. KEGG ko terms (column A), the number of differentially expressed genes in each ko category (B), the number of genes in each ko category (C), $P$ value (D), annotations (E-G) and gene ID $(H)$ are shown.

Additional file 6: List of designed primers for quantitative RT-PCR.

\section{Competing interests}

The authors declare that they have no competing interests.

\section{Authors' contributions}

YJY, XCW, QYZ and XL conceived and designed the experimental plan. XCW, $C L M, H L C, C Y, X Y H, L C, J Q M$ and JQJ participated in sample collection and performed experiments. QYZ, XCW, ZHZ and YMK performed bioinformatics analyses. XCW and QYZ deposited the sequencing data in the GenBank and SRA databases. XCW, QYZ, XL and YJY drafted and revised the manuscript. All authors read and approved the final manuscript.

\section{Acknowledgements}

We thank Dr. Enda Byrne and Miss Emily Wheeler for discussions and critical reading of the manuscript. This work was supported by the National Natural Science Foundation of China (31170650, 31100504), the Natural Science Foundation of Zhejiang Province (Z3100473), the Earmarked Fund for China Agriculture Research System (CARS-23), the National Key Technology Research and Development Program of the Ministry of Science and Technology of China (2011BAD01B01-ZCS) and the National Key Basic Research Program in China (2013CB127005).

\section{Author details}

${ }^{1}$ Tea Research Institute, Chinese Academy of Agricultural Sciences; National Center for Tea Improvement, Key Laboratory of Tea Plant Biology and Resources Utilization, Ministry of Agriculture, Hangzhou 310008, China. ${ }^{2}$ Key Laboratory of Synthetic Biology, Institute of Plant Physiology and Ecology, Shanghai Institutes for Biological Sciences Chinese Academy of Sciences, Shanghai 200032, China. ${ }^{3}$ The University of Queensland, Queensland Brain Institute, Qld 4072, Australia.

Received: 22 December 2012 Accepted: 18 June 2013

Published: 22 June 2013

\section{References}

1. Thomashow MF: Plant cold acclimation: freezing tolerance genes and regulatory mechanisms. Annu Rev Plant Physiol Plant Mol Biol 1999, 50:571-599.

2. Hare PD, Cress WA, Van Staden J: Dissecting the roles of osmolyte accumulation during stress. Plant Cell Environ 1998, 21(6):535-553.

3. Iba K: Acclimative response to temperature stress in higher plants: approaches of gene engineering for temperature tolerance. Annu Rev Plant Biol 2002, 53:225-245

4. Atici O, Nalbantoglu B: Antifreeze proteins in higher plants. Phytochemistry 2003, 64(7):1187-1196.

5. Wang YJ, Zhang ZG, He XJ, Zhou HL, Wen YX, Dai JX, Zhang JS, Chen SY: A rice transcription factor OsbHLH1 is involved in cold stress response. Theor Appl Genet 2003, 107(8):1402-1409.

6. Gusta LV, Wisniewski M, Nesbitt NT, Gusta ML: The effect of water, sugars, and proteins on the pattern of ice nucleation and propagation in acclimated and nonacclimated canola leaves. Plant Physiol 2004, 135(3):1642-1653.

7. Chen THH, Murata N: Glycinebetaine: an effective protectant against abiotic stress in plants. Trends Plant Sci 2008, 13(9):499-505.

8. Fowler S, Thomashow MF: Arabidopsis transcriptome profiling indicates that multiple regulatory pathways are activated during cold acclimation in addition to the CBF cold response pathway. Plant Cell 2002, 14(8):1675-1690.

9. Chen L, Zhou Z-X, Yang Y-J: Genetic improvement and breeding of tea plant (Camellia sinensis) in China: from individual selection to hybridization and molecular breeding. Euphytica 2007, 154:239-248.

10. Wang Y, Jiang C-J, Li Y-Y, Wei C-L, Deng W-W: CsICE1 and CsCBF1: two reanscription factors involved in cold responses in Camellia sinenesis. Plant Cell Rep 2012, 31(1):27-34.

11. Yang YJ, Zheng LY, Wang XC: Effect of cold acclimation and ABA on cold hardiness, contents of proline of tea plants [Camellia sinensis $(\mathrm{L}$.) $\mathrm{O}$. Kuntze]. J Tea Sci 2004, 24(3):177-182. 
12. Wang $X C$, Yang YJ: Research progress on resistance breeding of tea plant. J Tea Sci 2003, 23(2):94-98.

13. Mei JF, Wang XC, Yang YJ, Li XH: Preliminary study on differential gene expression during cold acclimation in tea plant (Camellia sinensis). J Tea Sci 2007, 27(4):286-292

14. Chen L, Chen J, Jiang Y, Zhang W, Jiang W, Lu Y: Transcriptomics analyses reveal global roles of the regulator Avel in Streptomyces avermitilis. FEMS Microbiol Lett 2009, 298(2):199-207.

15. Sanghera GS, Wani SH, Hussain W, Singh NB: Engineering cold stress tolerance in crop plants. Curr Genomics 2011, 12(1):30-43.

16. Wang Z, Gerstein M, Snyder M: RNA-Seq: a revolutionary tool for transcriptomics. Nat Rev Genet 2009, 10(1):57-63.

17. Marguerat S, Bahler J: RNA-seq: from technology to biology. Cell Mol Life Sci 2010, 67(4):569-579.

18. Audic S, Claverie JM: The significance of digital gene expression profiles. Genome Res 1997, 7(10):986-995.

19. Zhao QY, Wang Y, Kong YM, Luo D, Li X, Hao P: Optimizing de novo transcriptome assembly from short-read RNA-Seq data: a comparative study. BMC Bioinforma 2011, 12(Suppl 14):S2

20. Grabherr MG, Haas BJ, Yassour M, Levin JZ, Thompson DA, Amit I, Adiconis $X$, Fan L, Raychowdhury R, Zeng Q, et al: Full-length transcriptome assembly from RNA-Seq data without a reference genome. Nat Biotechnol 2011.

21. Shi CY, Yang H, Wei CL, Yu O, Zhang ZZ, Jiang CJ, Sun J, Li YY, Chen Q, Xia $T$, et al: Deep sequencing of the Camellia sinensis transcriptome revealed candidate genes for major metabolic pathways of tea-specific compounds. BMC Genomics 2011, 12:131.

22. Wu CH, Apweiler R, Bairoch A, Natale DA, Barker WC, Boeckmann B, Ferro S, Gasteiger $E_{1}$ Huang $H$, Lopez $R$, et al: The universal protein resource (UniProt): an expanding universe of protein information. Nucleic Acids Res 2006, 34(Database issue):D187-191.

23. Kanehisa M, Goto S, Kawashima S, Okuno Y, Hattori M: The KEGG resource for deciphering the genome. Nucleic Acids Res 2004, 32(Database issue): D277-280

24. Tatusov RL, Fedorova ND, Jackson JD, Jacobs AR, Kiryutin B, Koonin EV, Krylov DM, Mazumder R, Mekhedov SL, Nikolskaya AN, et al: The COG database: an updated version includes eukaryotes. BMC Bioinforma 2003, 4:41.

25. Conesa A, Gotz S, Garcia-Gomez JM, Terol J, Talon M, Robles M: Blast2GO: a universal tool for annotation, visualization and analysis in functional genomics research. Bioinformatics 2005, 21(18):3674-3676.

26. OToole N, Hattori M, Andres C, lida K, Lurin C, Schmitz-Linneweber C, Sugita M, Small I: On the expansion of the pentatricopeptide repeat gene family in plants. Mol Biol Evol 2008, 25(6):1120-1128.

27. Li B, Dewey CN: RSEM: accurate transcript quantification from RNA-Seq data with or without a reference genome. BMC Bioinforma 2011, 12:323.

28. Anders S, Huber W: Differential expression analysis for sequence count data. Genome Biol 2010, 11(10):R106.

29. Janská A, Marík P, Zelenková S, Ovesná J: Cold stress and acclimation - what is important for metabolic adjustment? Plant Bio/ 2010, 12(3):395-405.

30. Reddy AS, Ali GS, Celesnik H, Day IS: Coping with stresses: roles of calcium- and calcium/calmodulin-regulated gene expression. Plant Cell 2011, 23(6):2010-2032

31. Vergnolle C, Vaultier M-N, Taconnat L, Renou J-P, Kader J-C, Zachowski A Ruelland E: The cold-induced early activation of phospholipase C and D pathways determines the response of Two distinct clusters of genes in Arabidopsis cell suspensions. Plant Physiol 2005, 139:1217-1233.

32. Munnik T: Phosphatidic acid: an emerging plant lipid second messenger. Trends Plant Sci 2001, 6(5):227-233.

33. Doherty CJ, Van Buskirk HA, Myers SJ, Thomashow MF: Roles for Arabidopsis CAMTA transcription factors in cold-regulated gene expression and freezing tolerance. Plant Cell 2009, 21:972-984

34. Yang T, Shad Ali G, Yang L, Du L, Reddy AS, Poovaiah BW: Calcium/ calmodulin-regulated receptor-like kinase CRLK1 interacts with MEKK1 in plants. Plant Signal Behav 2010, 5(8):991-994.

35. Chinnusamy V, Zhu JK, Sunkar R: Gene regulation during cold stress acclimation in plants. Methods Mol Biol 2010, 639:39-55.

36. Theocharis A, Clement C, Barka EA: Physiological and molecular changes in plants grown at low temperatures. Planta 2012, 235(6):1091-1105.

37. Lehti-Shiu MD, Zou C, Hanada K, Shiu SH: Evolutionary history and stress regulation of plant receptor-like kinase/pelle genes. Plant Physiol 2009, 150(1):12-26
38. Osakabe Y, Yamaguchi-Shinozaki K, Shinozaki K, Tran LSP: Sensing the environment: key roles of membrane-localized kinases in plant perception and response to abiotic stress. J Exp Bot 2013, 64(2):445-458.

39. Suzuki I, Los DA, Kanesaki Y, Mikami K, Murata N: The pathway for perception and transcription of low-temperature signals in Synechocystis. EMBO J 2000, 19:1327-1334.

40. Jeon J, Kim NY, Kim S, Kang NY, Novak O, Ku SJ, Cho C, Lee DJ, Lee EJ, Strnad $M$, et al: A subset of cytokinin two-component signaling system plays a role in cold temperature stress response in Arabidopsis. J Biol Chem 2010, 285(30):23371-23386.

41. van Buskirk HA, Thomashow MF: Arabidopsis transcription factors regulating cold acclimation. Physiol Plant 2006, 126(1):72-80.

42. Toledo-Ortiz G, Huq E, Quail PH: The Arabidopsis basic/helix-loop-helix transcription factor family. Plant Cell 2003, 15(8):1749-1770.

43. Rushton PJ, Somssich IE, Ringler P, Shen QJ: WRKY transcription factors. Trends Plant Sci 2010, 15(5):247-258

44. Zhang L, Zhao G, Jia J, Liu X, Kong X: Molecular characterization of 60 isolated wheat MYB genes and analysis of their expression during abiotic stress. J Exp Bot 2012, 63(1):203-214

45. Jakoby M, Weisshaar B, Droge-Laser W, Vicente-Carbajosa J, Tiedemann J, Kroj T, Parcy F: bZIP transcription factors in Arabidopsis. Trends Plant SC 2002, 7(3):106-111

46. Hu H, You J, Fang Y, Zhu X, Qi Z, Xiong L: Characterization of transcription factor gene SNAC2 conferring cold and salt tolerance in rice. Plant $\mathrm{Mol}$ Biol 2008, 67(1-2):169-181.

47. Nishizawa A, Yabuta Y, Yoshida E, Maruta T, Yoshimura K, Shigeoka S: Arabidopsis heat shock transcription factor $\mathrm{A} 2$ as a key regulator in response to several types of environmental stress. Plant J 2006, 48(4):535-547.

48. Fode B, Siemsen T, Thurow C, Weigel R, Gatz C: The Arabidopsis GRAS protein SCL14 interacts with class II TGA transcription factors and is essential for the activation of stress-inducible promoters. Plant Cell 2008, 20:3122-3135.

49. Mukhopadhyay A, Vij S, Tyagi AK: Overexpression of a zinc-finger protein gene from rice confers tolerance to cold, dehydration, and salt stress in transgenic tobacco. Proc Natl Acad Sci U S A 2004, 101(16):6309-6314.

50. Catalá R, Medina J, Salinas J: Integration of low temperature and light signaling during cold acclimation response in Arabidopsis. Proc Natl Acad Sci U S A 2011, 108(39):16475-16480.

51. Jurczyk B, Rapacz M, Budzisz K, Barcik W, Sasal M: The effects of cold, light and time of day during low-temperature shift on the expression of CBF6, FpCor14b and LOS2 in Festuca pratensis. Plant Sci 2012, 183:143-148.

52. Conde A, Chaves MM, Geros H: Membrane transport, sensing and signaling in plant adaptation to environmental stress. Plant Cell Physiol 2011, 52(9):1583-1602.

53. Zhou Z, Wang M-J, Zhao S-T, Hu J-J, Lu M-Z: Changes in freezing tolerance in hybrid poplar caused by up- and down-regulation of PtFAD2 gene expression. Transgenic Res 2010, 19(4):647-654.

54. Li B, Takahashi D, Kawamura Y, Uemura M: Comparison of plasma membrane proteomic changes of Arabidopsis suspension-cultured cells (T87 Line) after cold and ABA treatment in association with freezing tolerance development. Plant Cell Physiol 2012, 53(3):543-554.

55. Upchurch RG: Fatty acid unsaturation, mobilization, and regulation in the response of plants to stress. Biotechnol Lett 2008, 30(6):967-977.

56. Kiełbowicz-Matuk A, Rey P, Rorat T: The organ-dependent abundance of a Solanum lipid transfer protein is up-regulated upon osmotic constraints and associated with cold acclimation ability. J Exp Bot 2008, 59(8):2191-2203.

57. Tall A: Plasma lipid transfer proteins. Annu Rev Biochem 1995, 64:235-257

58. Griffith M, Yaish MWF: Antifreeze proteins in overwintering plants: a tale of two activities. Trends Plant Sci 2004, 9(8):399-405.

59. Griffith M, Brown GN, Huner NP: Structural changes in thylakoid proteins during cold acclimation and freezing of winter rye (secale cereale L. Cv. Puma). Plant Physiol 1982, 70(2):418-423.

60. Gao MJ, Schafer UA, Parkin IA, Hegedus DD, Lydiate DJ, Hannoufa A: A novel protein from Brassica napus has a putative KID domain and responds to low temperature. Plant J 2003, 33(6):1073-1086.

61. Uemura M, Tominaga Y, Nakagawara C, Shigematsu S, Minami A, Kawamura $Y$ : Responses of the plasma membrane to low temperatures. Physiol Plant 2006, 126(1):81-89.

62. Usadel B, Blasing OE, Gibon Y, Poree F, Hohne M, Gunter M, Trethewey R, Kamlage B, Poorter $\mathrm{H}$, Stitt M: Multilevel genomic analysis of the response of transcripts, enzyme activities and metabolites in Arabidopsis rosettes to a progressive decrease of temperature in the non-freezing range. Plant Cell Environ 2008, 31(4):518-547. 
63. Oono Y, Seki M, Satou M, lida K, Akiyama K, Sakurai T, Fujita M, Yamaguchi-Shinozaki K, Shinozaki K: Monitoring expression profiles of Arabidopsis genes during cold acclimation and deacclimation using DNA microarrays. Funct Integr Genomics 2006, 6(3):212-234.

64. Wormit A, Trentmann O, Feifer I, Lohr C, Tjaden J, Meyer S, Schmidt U, Martinoia E, Neuhaus HE: Molecular identification and physiological characterization of a novel monosaccharide transporter from Arabidopsis involved in vacuolar sugar transport. Plant Cell 2006, 18(12):3476-3490.

65. Maruyama K, Takeda M, Kidokoro S, Yamada K, Sakuma Y, Urano K, Fujita M, Yoshiwara K, Matsukura S, Morishita Y, et al: Metabolic pathways involved in cold acclimation identified by integrated analysis of metabolites and transcripts regulated by DREB1A and DREB2A. Plant Physiol 2009, 150(4):1972-1980.

66. Nägele T, Kandel BA, Frana S, Meißner M, Heyer AG: A systems biology approach for the analysis of carbohydrate dynamics during acclimation to low temperature in Arabidopsis thaliana. FEBS J 2011, 278(3):506-518.

67. Fernandez $\mathrm{O}$, Theocharis $\mathrm{A}$, Bordiec $\mathrm{S}$, Feil $\mathrm{R}$, Jacquens $\mathrm{L}$, Clement $\mathrm{C}$, Fontaine F, Barka EA: Burkholderia phytofirmans PsJN acclimates grapevine to cold by modulating carbohydrate metabolism. Mol Plant Microbe Interact 2012, 25(4):496-504.

68. Livingston DP, Premakumar R, Tallury SP: Carbohydrate partitioning between upper and lower regions of the crown in oat and rye during cold acclimation and freezing. Cryobiology 2006, 52:200-208.

69. Palonen P, Buszard D, Donnelly D: Changes in carbohydrates and freezing tolerance during cold acclimation of red raspberry cultivars grown in vitro and in vivo. Physiol Plant 2000, 110(3):393-401.

70. Knight H, Trewavas AJ, Knight MR: Cold calcium signaling in Arabidopsis involves two cellular pools and a change in calcium signature after acclimation. Plant Cell 1996, 8(3):489-503.

71. Wang $\mathrm{Y}, \mathrm{Li} J$, Wang J, Li Z: Exogenous $\mathrm{H}_{2} \mathrm{O}_{2}$ improves the chilling tolerance of manilagrass and mascarenegrass by activating the antioxidative system. Plant Growth Regul 2010, 61(2):195-204.

72. Li W, Godzik A: Cd-hit: a fast program for clustering and comparing large sets of protein or nucleotide sequences. Bioinformatics 2006, 22(13):1658-1659.

73. Ashburner M, Ball CA, Blake JA, Botstein D, Butler H, Cherry JM, Davis AP, Dolinski K, Dwight SS, Eppig JT, et al: Gene ontology: tool for the unification of biology. The Gene Ontology Consortium. Nat Genet 2000, 25(1):25-29.

74. Finn RD, Mistry J, Tate J, Coggill P, Heger A, Pollington JE, Gavin OL, Gunasekaran P, Ceric G, Forslund K, et al: The Pfam protein families database. Nucleic Acids Res 2010, 38(Database issue):D211-222.

75. Yeats C, Redfern OC, Orengo C: A fast and automated solution for accurately resolving protein domain architectures. Bioinformatics 2010, 26(6):745-751.

76. Langmead B, Trapnell C, Pop M, Salzberg SL: Ultrafast and memory-efficient alignment of short DNA sequences to the human genome. Genome Biol 2009, 10(3):R25.

77. Gohain B, Bandyopadhyay T, Borchetia S, Bharalee R, Gupta S, Bhorali P, Agarwala N, Das S: Identification and validation of stable reference genes in Camellia Species. Journal of Biotechnology and Pharmaceutical Research 2011, 2(1):9-18.

78. Livaka K, Schmittgen TD: Analysis of relative gene expression data using real-time quantitative PCR and the $2^{-\Delta \Delta C T}$ method. Methods 2001, 25(4):402-408.

doi:10.1186/1471-2164-14-415

Cite this article as: Wang et al:: Global transcriptome profiles of Camellia sinensis during cold acclimation. BMC Genomics 2013 14:415.

\section{Submit your next manuscript to BioMed Central and take full advantage of:}

- Convenient online submission

- Thorough peer review

- No space constraints or color figure charges

- Immediate publication on acceptance

- Inclusion in PubMed, CAS, Scopus and Google Scholar

- Research which is freely available for redistribution

Submit your manuscript at www.biomedcentral.com/submit 